\title{
Editorial
}

\section{¿Se pueden explicar y modificar las diferencias en la calidad de vida percibida, entre pacientes incidentes y prevalentes en tratamiento de diálisis?}

\author{
Can you explain and modify the differences in the perceived quality of life, between patients incident and \\ prevalent in dialysis treatment?
}

En la literatura médica, con frecuencia, se encuentran publicaciones que hacen referencia a la calidad de vida de los pacientes en diálisis. Sin embargo, no es habitual hallar un trabajo que demuestre, con fortaleza estadística, la diferencia en la percepción de la calidad de vida entre los pacientes incidentes, que están cursando los primeros tres meses de su tratamiento hemodialítico, y prevalentes, que han superado este plazo, en muchos casos con periodos prolongados. Esta diferencia, precisamente, se ha logrado demostrar en el esclarecedor artículo producido por Varela y colegas, titulado "Calidad de vida en pacientes incidentes vs prevalentes. ¿Hay diferencia en la calidad de vida?”.

Así, surge con fuerza la necesidad de encontrar explicaciones que posibiliten, en primera instancia, comprender el fenómeno y, posteriormente, actuar y brindar las soluciones sanitarias que permitan modificar tales resultados. Para ello, resulta atinado recordar el camino histórico y evolutivo logrado por la humanidad en el control de las enfermedades y confrontarlo, en términos generales, con la calidad de vida alcanzada por las diferentes masas poblacionales. Fundamentalmente, en las regiones más desprotegidas del globo y, especialmente, en numerosas regiones de América Latina y el Caribe.

Sabemos que la medicina y los tratamientos médicos han evolucionado a través de los siglos y transformado rudimentarias técnicas de tratamiento, de origen artesanal, en los grandes logros terapéuticos que el conocimiento científico ha incorporado. Estos siguen transformando y ampliando la expectativa de vida del ser humano también hoy, a inicios del siglo XXI ${ }^{1,2}$.

Continuamente asistimos, absortos, a nuevos desarrollos de la farmacología y de la investigación biotecnológica, cuyos tenues límites imponen, necesariamente, la imprescindible contención de la bioética. Simultáneamente, se controlan epidemias, se mejora el control de enfermedades, se prolonga la expectativa de vida pero crecen exponencialmente, también, las enfermedades y los enfermos crónicos, entre ellos, los pacientes con enfermedad renal crónica.

Paradójicamente, estas novedosas situaciones pusieron de manifiesto la inequidad propia del mundo actual. En particular, las profundas diferencias de acceso y calidad de vida entre aquellas poblaciones con adecuados estándares de vida, habitantes legales de países y/o regiones desarrolladas, frente a las grandes masas poblacionales que viven en condiciones de vulnerabilidad sanitaria, o incluso de pobreza extrema, suponen diferencias en el acceso al cuidado, incluyendo la ausencia de estrategias educativas de promoción y prevención de las enfermedades, así como el autocuidado para el restablecimiento, recuperación y rehabilitación de la salud.

La ausencia de infraestructura accesible condiciona a las comunidades a llegar tardíamente al cuidado de la salud. Sin embargo, quienes hemos trabajado en terreno durante muchos años hemos podido apreciar que, aún estando disponible el centro de salud, la barreras más sólida al acceso al sistema sanitario está constituida por la ignorancia, las carencias educativas severas y, muchas veces, los modelos culturales de algunas zonas de Latinoamérica y del Caribe, donde se recurre a 'brujos', 'curanderos' y similares ${ }^{3-5 .}$ 
Latinoamérica, a nivel regional, es un claro exponente de estas grandes diferencias, que se agigantan cuando, dentro de los territorios nacionales, existen concentraciones de población con enormes diferencias distributivas en los recursos disponibles. En situaciones semejantes, la sociedad se enfrenta al dilema de la asignación de recursos económicos, habitualmente escasos en los países menos desarrollados.

La enfermedad renal crónica tiene un efecto negativo sobre la calidad de vida, la economía de las familias y de los sistemas de salud, dependiendo de la velocidad de progresión de la pérdida de la función renal. Una progresión rápida genera aumento de los costos, reducida calidad de vida y muerte prematura. La mortalidad en el estadio 4 (tasa de filtración glomerular de 30-15 ml/minuto) es superior al $45 \%$, que se suma a las muertes ocurridas en las etapas anteriores de la enfermedad, casi siempre por causas cardiovasculares. Los sobrevivientes, el porcentaje restante del estadio 4, ingresarán a diálisis, habitualmente en situaciones de emergencia y/o descompensación clínica, sin controles previos anticipatorios ni preparatorios para la terapéutica sustitutiva, cualquiera que sea la elegida. Se requirien, en tales casos, técnicas terapéuticas de salvataje, como la colocación de catéteres transitorios para su hemodiálisis de urgencia. Con ello se explica, suficientemente, la diferencia en calidad de vida y sobrevida de estos pacientes incidentes, de gran labilidad clínica y sin la adecuada preparación psicológica ni para su enfermedad ni para esta nueva situación de dependencia a los procesos tecnológicos como soporte vital de su existencia.

El paciente se enfrenta con un diagnóstico de insuficiencia renal crónica terminal, terminología que, aún, se continúa empleando en la literatura y que conlleva en sí misma una carga negativa adicional. Las palabras son las mediadoras, por excelencia, en nuestra vida social. Vemos su eficacia ya sea para acercarnos o alejarnos de personas o de situaciones. Algunas palabras nos confortan y otras nos aterran, según la persona, el modo y el contexto en que son pronunciadas. Cuando un médico le informa a su paciente que tiene insuficiencia renal terminal, la primera asociación con la palabra terminal suele ser lo que acaba, el final, la cercanía de la muerte. El Diccionario de la Real Academia Española dice de la palabra terminal: "dicho de un enfermo o de un paciente: que está en situación grave e irreversible y cuya muerte se prevé muy próxima"?

En este contexto, resulta fácil comprender que la calidad de vida, como concepto holístico y absolutamente subjetivo, no puede ser comparable entre personas provenientes de diferentes etnias culturales, países y grupos de población, con sus condiciones particulares. Aun dentro del mismo país, existen asimetrías.

Considero importante reafirmar conceptualmente que es un error, en el lenguaje, declarar terminal a un paciente cuando cursa etapas avanzadas de su enfermedad renal. Resulta aconsejable, entonces, modificar en la nomenclatura médica el concepto de insuficiencia renal crónica terminal para los pacientes en estadio 5 D. ${ }^{8}$, y transformarlo por insuficiencia renal crónica permanente. Con ello se generaría, también, una externación positiva dentro de los financiadores de salud, pues lo permanente deja de ser terminal.

Cuando los pacientes están controlados tempranamente y pueden lograr la regresión y/o remisión de la enfermedad renal, comienzan a modificarse las alternativas de descontrol global de la homeostasis, en sus aspectos biológicos y emocionales. Así, se impacta en la percepción subjetiva de la calidad de vida, más allá de la objetividad de los valores clínico-químicos alterados. Se superan, además, esquemas propios de la ausencia de programas sanitarios sistematizados y articulados con la sociedad, bajo el concepto de transdisciplina, con profesionales de la Salud comprometidos con su desarrollo. 
Por lo tanto, es necesario revisar, en cada país, las políticas de financiamiento de los programas sanitarios y de las macro-asignaciones presupuestarias secundarias a grupos diferenciados de población con necesidades de cobertura específica. Por ejemplo, en los programas articulados entre salud renal y otras patologías crónicas prevalentes, con una conceptualización holística e integral ${ }^{9}$.

Latinoamérica, a través de la Sociedad Latinoamericana de Nefrología e Hipertensión, ha sido pionera en propender e impulsar estas políticas públicas, suministrando evidencias de la necesidad e importancia de actuar integralmente en la prevención y control de las enfermedades renales ${ }^{10-12}$. Estos esfuerzos aún no se han cristalizado ni incluido en las políticas públicas de los países, con el resultado de una menor calidad de vida en los pacientes incidentes que en los prevalentes. No obstante, a primera vista, se esperarían mejores resultados, como el ingreso de pacientes bien compensados, controlados, con accesos vasculares permanentes permeables y listos para ser usados. Al menos, en su defecto, preparados para iniciar una terapia de diálisis peritoneal o incluso un trasplante renal, en los países en los cuales la legislación lo habilita.

Nuestras consideraciones estarían incompletas si no se destacara la importancia del rol educativo del equipo de salud. Ya es tiempo de que los nuevos modelos formativos, todavía centrados en un modelo biomédico, se enfoquen en una comprensión biomédica, ética, psicosocial y económica. Es preciso una concepción global, que busque lograr y mantener la calidad de vida, mediante la gestión y estímulo del conocimiento impartido y, fundamentalmente, la preocupación por la excelencia en la calidad de la práctica. Este último aspecto debe extenderse, necesariamente, hacia la calidad en las relaciones interpersonales, bien sean médico/paciente, médico/familia, o médico/comunidad. Asimismo, la enseñanza de las competencias genéricas transversales se debe incorporar en los currículos universitarios la enseñanza ${ }^{13}$.

Volvamos, en este punto, a la pregunta inicial: ¿se pueden explicar y modificar las diferencias en la calidad de vida percibida, entre pacientes incidentes y prevalentes en tratamiento de diálisis? Hemos visto, en esta exposición, los condicionantes que justifican tal diferencia. Sin embargo, lo más importante es señalar que, efectivamente, cuando se diseñan y ejecutan programas sistematizados se pueden modificar estas diferencias. Es el caso de lo publicado, en Colombia, por el Dr. Gustavo José Aroca Martinez, con un modelo regional de gestión de riesgo para la nefritis lúpica, pero asimilable a todas las patologías renales, en su dinámica de trabajo transdisciplinario ${ }^{14}$.

Santos Depine

Profesor emérito, Universidad del Salvador, Argentina Profesor investigador, Universidad Simón Bolívar, Colombia Coordinador internacional de RISRECP Director de Calidad de la Confederación de Asociaciones de Diálisis de la República Argentina. Asesor para el Análisis y Evaluación de los Proyectos, Unidad de Financiamiento Internacional, Ministerio de Salud, Argentina adepine@intramed.net 


\section{Referencias}

1. Depine S. El médico en la consulta. La rejerarquización académica de viejas artesanías. Drogas. Mejor hablar de ciertas cosas. 1997. Facultad de Derecho. Univ. Nac. de Bs. As.

2. Challú A, Burgos-Calderón R, Depine, S, Feler, D, \& Manzor, D. (1999). La Nefrología en Latinoamérica. . Buenos Aires: SLANH.

3. Plata R, Silva C, Yahuita J, Perez L, Schieppati A, Remuzzi G. The first clinical and epidemiological program on renal disease in Bolivia: a model for prevention and early diagnosis of renal diseases in the developing countries. Nephrol Dial Transplant. 1998;13:3034-3036

4. Depine S. Programa nacional de prevención y control de enfermedades renales. Ministerio de Salud y Deportes. República de Bolivia. 2007. Disponible en: http://www.fundacionvidasaludable.org/Docs/RenalHealthProgram/Prog_Prevenci $\%$ C3\%B3n_\%20 Control_Enf_Renales_Bolivia.pdf

5. Ministerio de Salud y Deportes. Programa nacional de prevención y control de enfermedades renales. Documentos técnicos normativos. Publicación 42. Bolivia. 2008

6. Keith DS, Nichols GA, Gullion CM, Brown JB, Smith DH. Longitudinal follow-up and outcomes among a population with chronic kidney disease in a large managed care organization. Arch Intern Med. 2004;164:659-63. Disponible en: https://doi. org/10.1001/archinte.164.6.659

7. Diccionario de la Real Academia Española. Disponible en: http://dle.rae.es

8. Depine S \& Burgos-Calderón R. La integración de la salud pública y la nefrología clínica. Una asignatura pendiente para el siglo XXI. 2007. Disponible en: http://www.fundacionvidasaludable.org/Docs/RenalHealthProgram/IntegracionEntreSaludPublica_y NefrologiaClinica.pdf

9. Depine S. The role of government and competing priorities in minority populations and developing nations. Ethnicity \& Disease. 2009;19:73-9. Disponible en: http://www.ishib.org/journal/19-1s1/ethn-19-01s1-73.pdf

10. Burgos-Calderón R \& Depine S. Sustainable and tenable renal health model: A Latin American proposal of classification, programming, and evaluation. Kidney Int. 2005;68(Supp 97):S23-30.

11. Depine S \& Burgos-Calderón R. Renal Health Models in Latin America: Development of National Programs of Renal Health. Ren. Fail. 2006;28(8):649-64. Disponible en: https://doi.org/10.1080/08860220600925743

12. Burgos-Calderón R \& Depine S. Systematic approach for the management of chronic kidney disease: Moving beyond CKD classification. Curr Opin Nephrol Hypertens. 2010;19(2).

13. Depine S. Gestión del conocimiento en las facultades de Medicina. Rev Colomb Nefrol. 2014;1(1):25-38. Disponible en: $\underline{\text { https:// }}$ doi.org/10.22265/acnef.1.1.170

14. Aroca-Martínez GJ. Propuesta de un modelo de gestión de salud de la nefritis lúpica basado en la problemática clínica y su impacto socio-sanitario en la región caribe colombiana. Grupo de Investigación Nefrología. Facultad Ciencias de la Salud. Universidad Simón Bolívar. 2017

140 ¿Se pueden explicar y modificar las diferencias en la calidad de vida percibida, entre pacientes incidentes y prevalentes en tratamiento de diálisis? 